\title{
THE
}

UNIVERSITY

University of Rhode Island

OF RHODE ISLAND

DigitalCommons@URI

4-22-1985

\section{Effect of Criticality on Wetting Layers}

\author{
M. P. Nightingale \\ University of Rhode Island, nightingale@uri.edu \\ J. O. Indekeu
}

Follow this and additional works at: https://digitalcommons.uri.edu/phys_facpubs

Terms of Use

All rights reserved under copyright.

\section{Citation/Publisher Attribution}

Nightingale, M. P., \& Indekeu, J. O. (1985). Effect of Criticality on Wetting Layers. Physical Review Letters, 54(16), 1824-1827. doi: 10.1103/PhysRevLett.54.1824

Available at: http://dx.doi.org/10.1103/PhysRevLett.54.1824

This Article is brought to you for free and open access by the Physics at DigitalCommons@URI. It has been accepted for inclusion in Physics Faculty Publications by an authorized administrator of DigitalCommons@URI. For more information, please contact digitalcommons-group@uri.edu. 


\title{
Effect of Criticality on Wetting Layers
}

\author{
M. P. Nightingale \\ Department of Physics, University of Rhode Island, Kingston, Rhode Island 02881, and Department of Physics, \\ Massachusetts Institute of Technology, Cambridge, Massachusetts 02139 \\ and \\ J. O. Indekeu \\ Department of Physics, Massachusetts Institute of Technology, Cambridge, Massachusetts 02139, and \\ Instituut voor Theoretische Fysica, Katholieke Universiteit Leuven, 3030 Leuven, Belgium $^{(\mathrm{a})}$ \\ (Received 21 June 1984)
}

\begin{abstract}
We study wetting phenomena in which the wetting layer is (nearly) critical and intrudes between two noncritical phases. Finite-size scaling theory predicts an interaction, identical in range to that due to the van der Waals forces, between the interfaces bounding the wetting layer. This finite-size interaction leads to new wetting phenomena near critical end points, e.g., in ternary mixtures. The interaction amplitude and its possible universality can be observed directly in experiment.
\end{abstract}

PACS numbers: 68.10.- m, 05.70.Jk, 64.70.-p, 75.40.Dy

In a container with two coexisting phases $\alpha$ and $\beta$, the $\alpha$ phase is said to (completely) wet the wall, if a macroscopically thick layer of $\alpha$ spontaneously intrudes between $\beta$ and the wall. Models ${ }^{1}$ and experiments ${ }^{2-4}$ both display this behavior and the corresponding phase transition from incomplete to complete wetting. Also, it has been argued that wetting quite generally occurs in the vicinity of critical points, where $\alpha$ and $\beta$ become identical. ${ }^{2}$ However, as argued by de Gennes, systems with long-range forces may provide exceptions to this general behavior. ${ }^{5}$ The interactions considered by de Gennes are microscopic, van der Waals forces. A long-range interaction of very different physical origin arises in nearly critical systems of finite size, due to the incipient long-ranged correlations. ${ }^{6}$ A remarkable coincidence is that both finitesize and van der Waals forces give rise to a contribution to the free energy of a wetting layer which depends on its thickness $z$ as $z^{-2}$ in three dimensions. The ranges of the forces being equal, we show in this Letter that also their relative magnitudes match in systems of experimental interest. One such example is a ternary mixture at a critical end point in the vicinity of the tricritical point. We suggest that new wetting and dewetting phenomena are associated with the interplay of microscopic and finite-size forces. Furthermore, a study of these behaviors will shed light on the intriguing problem of the universality of the finite-size amplitude.?

We consider a system with a tricritical point, and assume that this point is approached along a line of critical end points. Then, one of the two phases, say the critical phase, will tend to wet the wall. That is, scaling arguments show that the total interface free energy is lowered by replacing the (nonequilibrium) interface of the wall and the noncritical spectator phase $\beta$ by a combination of a wall- $\alpha$ and an $\alpha-\beta$ interface infinitely far apart (complete wetting). Long-range forces result in a net interaction, which acts on the latter interfaces and which decays algebraically with their separation $z$. If this interaction is attractive, the free energy is reduced even further when the wall- $\alpha$ and the $\alpha-\beta$ interfaces have a finite rather than an infinite separation: Incomplete wetting is the result. Namely, the decrease in free energy associated with the long-range tails is of order $z^{-2}$. The distortion in the presence of the wall of the central region of the $\alpha-\beta$ interface, on the other hand, leads to an increase in free energy of order $e^{-z / \xi}$, where $\xi$, the correlation length, is a measure for the extent of that region. The study of the form of the compound interface which results from this delicate balance is beyond the scope of this Letter. Focusing solely on the question of complete or incomplete wetting, we shall make a number of plausible assumptions which allow us to estimate the magnitude of the finite-size interaction between the interfaces that bound the wetting layer, and compare with the van der Waals forces.

First, all interfaces are assumed to be rigid. In suppressing fluctuations in the boundary geometry, a term in the interfacial free energy that is exponentially small in the layer thickness in three (but not in two) dimensions ${ }^{8}$ is neglected.

Second, the free energy is assumed to be the sum of two terms. One is a term that takes into account the long-range part of the microscopic forces. The other pertains to the remainder, an effective system with short-range forces only. The possiblity of such a separation is based on the notion that the long-range nature of van der Waals forces is irrelevant, in the renormalization-group sense. ${ }^{9}$ Although this irrelevance was established for bulk critical behavior only, it can be consistently extended to surface (or interface) phenomena, as follows. Bonds broken due to 
the presence of a surface give rise to an ordering surface field $h_{l r}$, that falls off into the bulk as $h_{l r} \sim l^{-\omega}, l$ being the distance to the surface $(\omega=3$ for van der Waals forces). If a bulk ordering field $h$ renormalizes to $b^{y_{h}} h$ under spatial rescaling by a factor of $b$, the scaling index of $h_{l r}$ is $y_{h}-\omega$. For $\omega>y_{h}-$ as is the case for van der Waals forces and continuous transitions in three dimensions - this field is irrelevant, in the sense that under renormalization it reduces to a short-range field, acting only at the boundary. Also, this line of reasoning makes the assumption of infinitely sharp interfaces plausible, as far as universal aspects of critical behavior are concerned.

Henceforth we assume that the van der Waals forces have been separated out, and we consider an effective system with short-range interactions only. To calculate typical amplitudes of the purely finite-size interface interaction of the (nearly) critical wetting layer we consider an Ising model on a $d$-dimensional hypercubic lattice: $z$ layers of $n$ by $n$. . $(d-1$ times $)$ sites each. The interactions are only nearest-neighbor and such that the $d$-dimensional bulk $(z, n \rightarrow \infty)$ is critical. The wall on one side and the spectator phase on the other are modeled by boundary magnetic fields $h_{1}$ and $h_{2}$, acting on the top and bottom layers only. The interaction between the two boundaries is given by

$$
\Delta f\left(z, h_{1}, h_{2}\right)=\lim _{n \rightarrow \infty}\left[F-z n^{d-1} f_{b}\right] / n^{d-1}-\left(f_{1}+f_{2}\right) .
$$

Here $F$ is the total free energy of the system, $f_{b}$ the bulk free energy per spin, and $f_{i}(i=1,2)$ the excess free energy per surface spin of a semi-infinite system with boundary field $h_{i}$. Subtracting $f_{1}+f_{2}$ makes $\Delta f$ vanish for $z \rightarrow \infty$. The effective interaction of $\Delta f$ satisfies the following finite-size scaling relation

$$
\Delta f\left(z, h_{1}, h_{2}\right)=b^{-(d-1)} \Delta f\left(\frac{z}{b}, b^{y_{h}^{\prime}} h_{1}, b^{y_{h}^{\prime}} h_{2}\right) .
$$

This relation was previously studied by Fisher and de Gennes in the context of a nearly critical fluid between two plates. ${ }^{6}$ For large $z$ (so that $z^{y_{h}^{\prime}}\left|h_{i}\right| \gg>1$ for $\left.h_{i} \neq 0\right)$ Eq. (1) yields $\Delta f / k T_{c} \simeq \Delta z^{d-1}$ where $\Delta$ can assume one of four values: $\Delta_{00}$ (if $h_{1}=h_{2}=0$ ), $\Delta_{0+}$ (if $h_{1} \neq h_{2}=0$ or $\left.h_{2} \neq h_{1}=0\right), \Delta_{++}$(if $\left.h_{1} h_{2}>0\right)$, and $\Delta_{+-}$(if $h_{1} h_{2}<0$ ), corresponding to the renormalization-group fixed points at $h_{i}=0, \pm \infty$. It is tempting to speculate that these four possible values of $\Delta$ are universal numbers. ${ }^{7}$ Universality with respect to the magnitude of the boundary fields, which is implied by Eq. (1), has been verified by exact calculations in two dimensions. The second aspect, universality throughout the whole Ising universality class, is more speculative. It does hold in the simplest, most appealing version of the theory to which, to our knowledge, there are no counterexamples as yet. ${ }^{10}$ Most impor- tantly, if universality holds in all its aspects, the finite Ising model represents a valid description for our purpose of the (nearly) critical wetting layer.

In very few cases amplitudes of finite-size forces between surfaces can be calculated exactly. For $d=1$, sufficiently close to $T=0$, exact calculation yields exponential attraction for $h_{1} h_{2}>0$. If $h_{1} h_{2}<0$, an exponential repulsion results. In both cases the force does not depend on the magnitude of the fields. There is no force for $h_{1} h_{2}=0$. For $d=2 \mathrm{Au}-$ Yang and Fisher ${ }^{11}$ found agreement with finite-size scaling, and obtained $\Delta_{00}=-\pi / 48$. Employing the same method, we derived $\Delta_{0+}=\pi / 24$, and $\Delta_{++}=-\pi / 48$ and explicitly verified the irrelevance of the magnitude of the surface fields. Following arguments by Au-Yang and Fisher (cf. also mean-field results below) we may further speculate that $\Delta_{+-}=2^{d} \Delta_{+0}=\pi / 6$.

We have estimated the strength of the interaction between the surfaces for $d=3$, with a renormalization group ( $R G$ ) calculation of the Migdal-Kadanoff type. ${ }^{12}$ We start out with a system of $z=b^{m}+1$ layers, where $m$ is integer and $b$, the rescaling length, is close to 1 . Renormalization generates a surface nearest-neighbor coupling different from that in the bulk. $m$ RG iterations reduce the slab to two layers $(z=2)$. At this point additional interactions (diagonal and four-spin) are introduced, and renormalization proceeds in a way which exactly solves the double chain $(d=2)$. The fixed points which yield the various finite-size amplitudes correspond to the bulk critical fixed point combined with the surface fixed points mentioned above. For $d=2$ we find $\Delta_{00}=-0.055(-0.065$ is exact $)$, $\Delta_{0+} \simeq 0.130(0.131$ is exact $), \Delta_{++}=0(-0.065$ is exact), and $\Delta_{+_{-}}=0.881(0.524$ is expected $)$. The agreement with the exact results in $d=2$ suggests that the estimates obtained for $d=3$, viz. $\Delta_{00} \simeq-0.015$, $\Delta_{0+} \simeq 0.051, \Delta_{++}=0$, and $\Delta_{+-} \simeq 0.279$ are reliable at least in order of magnitude.

Finally, in a mean-field approximation the equilibrium density profile at criticality $m(x), 0 \leqslant x \leqslant z$, minimizes the total reduced free energy functional per unit area

$$
\begin{aligned}
F=\int_{0}^{z}\left[\frac{1}{4} u m^{4}\right. & \left.+\frac{1}{2} c(d m / d x)^{2}\right] d x \\
& +\frac{1}{2} v\left[m(0)^{2}+m(z)^{2}\right] \\
& -h_{1} m(0)-h_{2} m(z) .
\end{aligned}
$$

Following Nakanishi and Fisher, we took $u=\frac{1}{5}$ and $c^{2}=1 / 2 d$, the surface interaction $v>0$ is arbitrary. ${ }^{13}$ We find $\Delta_{00}=0, \Delta_{++}=-\frac{1}{12}\left(c^{2} / u\right) K^{4} \simeq-2.461 / d$, and $\Delta_{+-}=\frac{16}{3}\left(c^{2} / u\right) K^{4} \simeq 157.5 / d$, where $K$ is the complete elliptic integral of the first kind of modular angle $\pi / 4$. The interface interaction decays as $z^{-3}$ for large $z$. The amplitudes do not depend on the magnitude of the boundary fields for the following reason. 
In terms of a well-known mechanical analog, ${ }^{14}$ the profile as a function of position corresponds to the position $(m)$ as a function of time $(x)$ of a particle of mass $c$ on a potential hill $-\frac{1}{4} u m^{4}$. The change $\delta F$ in $F$ with a change in thickness $\delta z$ is given by $\delta F=-E \delta z$, where $E$ is the energy of the particle. The particle needs a finite time only (i.e., finite thickness $z$ ) to go from $m=-\infty$ to $m=\infty$ (or vice versa) if $E=3 \Delta_{+}$ $\times z^{-4}>0$, or from $m=- \pm \infty$ to close to the top of the hill and back if $E=3 \Delta_{++} z^{-4}<0$. This corresponds to infinite boundary fields. A fixed $E$ and finite $h_{i}$ in the range $\left|h_{i}\right| z>>v(2 c / u)^{1 / 2}$ yield values of $z$ with relative variations of order $v(2 c / u)^{1 / 2}$ only, which explains the universality in $h_{i}$. Also, it is found that the profile for a system of size $z$ with $h_{1} h_{2}<0$ is identical to the profile for a system of size $\frac{1}{2} z+O(c / v)$ and $h_{1} \neq h_{2}=0$, so that $\Delta_{0+}=2^{-4} \Delta_{+}$.

The results above suggest that, in general, like boundaries $\left(h_{1} h_{2}>0\right.$ or $\left.h_{1}=h_{2}=0\right)$ attract, but unlike boundaries ( $h_{1} h_{2}<0$ or one zero field) repel. The lack of interaction in some cases presumably is a peculiarity of $d=1$, mean-field theory, and the MigdalKadanoff RG approximation.

We now proceed to apply these results to systems of experimental interest. Having estimated the amplitudes due solely to finite-size effects, we now compare with the complementary case of van der Waals forces only. At large separations $z$ of the interfaces, the interaction free energy per unit area decays as $z^{-2}$ for both forces in three dimensions. Measured in units of $k T$ the respective amplitudes are $\Delta$ and $W$. Measurements on binary liquids by Kwon et al. yield $W \leq 0.01 .^{4}$ This value, which we take to be typical of fluid mixtures, is comparable to the value of $\Delta$ obtained above in $d=3$. This suggests that the interplay between both effects should be observable at critical end points in the vicinity of the tricritical point in ternary, or more-component, mixtures. As a matter of fact, the magnitude of the finite-size effect relative to the van der Waals interaction, $|\Delta / W|$, increases as one approaches the tricritical temperature $T_{\mathrm{tc}}$. Indeed, $W$ will go to zero, proportional to the difference in densities of the critical phase $\alpha$ and the spectator phase $\beta$, i.e, proportional to $\left|T-T_{\mathrm{tc}}\right|^{1 / 2} .^{15}$ On the other hand, the finite-size force depends only on the relative signs of the effective boundary fields and therefore $\Delta$ remains unchanged, if universality holds. In principle this can be exploited to measure $\Delta$ and test its speculated universality. Suppose that, as in the experimental setup, e.g. described by Kwon et al., ${ }^{4}$ a layer of thickness $z$ of the denser, but now supposedly critical, phase $\alpha$ (of density $\rho_{\alpha}$ ) is in equilibrium above a $\beta$ layer of thickness $L$ and density $\rho_{\beta}<\rho_{\alpha}$. Write $W=w \Delta \rho$, with $\Delta \rho=\rho_{\alpha}-\rho_{\beta} ; w$ is constant near $T_{\text {tc }}$. Along the line of critical end ponts, for repulsive net forces $(\Delta+W>0)$ we find $z^{3}=2 k T(\Delta / \Delta \rho+w) / L g$, where $g$ is the gravitational acceleration, extending de Gennes' result. ${ }^{5}$ Close to $T_{\text {tc }}, z^{3}$ is linear in $\Delta \rho^{-1}$ with slope proportional to $\Delta$.

Various new wetting and dewetting phenomena arise naturally and should be experimentally observable: For a repulsive (attractive) finite-size force the layer thickness is maximal (minimal) at a critical end point, and this gets more pronounced closer to $T_{\text {tc }}$. Collapse of the layer results whenever $\Delta+W<0$. Note that only those thicknesses $z$ are well defined that exceed the correlation length in the spectator phase, which diverges as $\left|T-T_{\mathrm{tc}}\right|^{-1}$. Also, for thin films deviations from linearity of $z^{3}$ in $\Delta \rho^{-1}$ will result as $\Delta$ develops a boundary field dependence for $\left|h_{i}\right| z^{y_{h}^{\prime}} \leq 1$ [cf. Eq. (1)].

The question which we finally address is how the crucial signs of the boundary fields $h_{i}$ can be determined for an actual physical system. This can be done in a straightforward way by studying, e.g., contact angles away from the critical end point, where $\alpha$ has phase separated. There the $h_{i}$, being associated with the noncritical wall and spectator phase, respectively, will have undergone slight modifications only.

In general, we expect the van der Waals and finitesize interactions to be comparable whenever the polarization densities of the $\alpha$ and $\beta$ phases are close. This is, for example, not the case for solid layers adsorbed on graphite, where $W$ is 2 or 3 orders of magnitude too big. ${ }^{16}$ Finally, the critical end points on the $\lambda$ line of liquid helium are interesting. Here, finite-size interactions outside the Ising universality class come into play.

Experimentally, if the van der Waals force can be arranged to be sufficiently small, an advantage of the $\lambda$ transition is that the gravitational field does not couple to the order parameter of the wetting layer. Since it does in general, in the case of the fluid mixtures, precautions have to be taken to ensure that the wetting layer has the critical composition, e.g., by the choice of special mixtures (such that phases of almost equal density, but different composition, become critical at the critical end point), or by setting up the experiment such that the thickness of the wetting layer equilibrates more rapidly (via a macroscopic flow) than its composition (via diffusion).

It is our pleasure to acknowledge valuable discussions with A. N. Berker, J. S. Desjardins, M. Kaufman, M. Moldover, M. Schick, J. M. J. van Leeuwen, and W. V. Wang. We thank M. E. Fisher for useful correspondence. One of us (J.O.I.) thanks Professor A. N. Berker for hospitality and support and acknowledges receipt of a Fulbright-Hays Award. This research was supported by the Joint Service Electronics Program under Contract No. DAAG29-83-K0003, by the National Science Foundation under Contract No. DMR-8406186, and by the Belgian National Fund 
for Scientific Research.

(a) Current address.

1J. W. Cahn, J. Chem. Phys. 66, 3667 (1977); E. Brézin, B. I. Halperin, and S. Leibler, Phys. Rev. Lett. 50, 1387 (1983); R. Lipowsky, D. M. Kroll, and R. K. P. Zia, Phys. Rev. B 27, 4499 (1983); E. H. Hauge and M. Schick, Phys. Rev. B 27, 4288 (1983), and references therein; P. Tarazona and R. Evans, Mol. Phys. 48, 799 (1983); P. Tarazona, M. M. Telo de Gama, and R. Evans, Mol. Phys. 49, 283 (1983); V. Privman, J. Chem. Phys. 81, 2463 (1984).

${ }^{2}$ M. R. Moldover and J. W. Cahn, Science 207, 1073 (1980); see Cahn, Ref. 1.

${ }^{3}$ D. W. Pohl and W. I. Goldburg, Phys. Rev. Lett. 48, 1111 (1982); J. W. Schmidt and M. R. Moldover, J. Chem. Phys. 79, 379 (1983).

${ }^{4} \mathrm{O}$ 'D. Kwon, D. Beaglehole, W. W. Webb, B. Widom,

J. W. Schmidt, J. W. Cahn, M. R. Moldover, and B. Stephenson, Phys. Rev. Lett. 48, 185 (1982).

5P.-G. de Gennes, J. Phys. (Paris) 42, L377 (1981).
${ }^{6}$ M. E. Fisher and P.-G. de Gennes, C.R. Acad. Sci. 287, 207 (1978).

${ }^{7}$ M. P. Nightingale and H. W. J. Blöte, J. Phys. A16, L657 (1983); V. Privman and M. E. Fisher, Phys. Rev. B 30, 322 (1984); J. L. Cardy, J. Phys. A 17, L385 (1984), and references therein.

${ }^{8}$ M. P. Nightingale, W. F. Saam, and M. Schick, Phys. Rev. Lett. 51, 1275 (1983).

${ }^{9}$ M. E. Fisher, S. K. Ma, and B. G. Nickel, Phys. Rev. Lett. 29, 917 (1972).

${ }^{10} \mathrm{H}$. W. J. Blöte and M. P. Nightingale [Physica (Utrecht) $112 \mathrm{~A}, 406$ (1982)] suggest the possibility $\Delta f \sim z^{1-d} \ln z$. Also in this case the amplitude is universal.

${ }^{11} \mathrm{H}$. Au-Yang and M. E. Fisher, Phys. Rev. B 11, 3469 (1975), and 21, 3956 (1980); M. E. Fisher and H. Au-Yang, Physica (Utrecht) 101A, 255 (1980).

${ }^{12}$ L. P. Kadanoff, Ann. Phys. (N.Y.) 100, 359 (1976).

${ }^{13}$ H. Nakanishi and M. E. Fisher, J. Chem. Phys. 78, 3279 (1983).

${ }^{14}$ E.g., B. Widom, J. Chem. Phys. 66, 872 (1977).

${ }^{15}$ R. B. Griffiths, J. Chem. Phys. 60, 195 (1974).

16J. L. Seguin, J. Suzanne, M. Bienfait, J. G. Dash, and J. A. Venables, Phys. Rev. Lett. 51, 122 (1983). 